\title{
Genetic correction of $\beta$-thalassemia patient-specific iPS cells and its use in improving hemoglobin production in irradiated SCID mice
}

Yixuan Wang ${ }^{1,}$, , Chen-Guang Zheng ${ }^{2, *}$, Yonghua Jiang ${ }^{1}$, Jiqin $Z_{\text {hang }}{ }^{1}$, Jiayu Chen ${ }^{1}$, Chao Yao ${ }^{1}$, Qingguo Zhao ${ }^{1}$, Sheng Liu ${ }^{1}, \mathrm{Ke} \mathrm{Chen}^{2}$, Juan $\mathrm{Du}^{2}, \mathrm{Ze} \mathrm{Yang}{ }^{3}$, Shaorong Gao ${ }^{1}$

${ }^{I}$ National Institute of Biological Sciences, Zhongguancun Life Science Park, \#7 Science Park Road, Beijing 102206, China; ${ }^{2}$ Guangxi Zhuang Autonomous Region Women and Children Care Hospital, Nanning, Guangxi 530003, China; ${ }^{3}$ National Institute of Geriatrics, Beijing Hospital, Chinese Ministry of Health, Beijing 100730, China

The generation of induced pluripotent stem cells (iPSCs) from differentiated somatic cells by over-expression of several transcription factors has the potential to cure many genetic and degenerative diseases currently recalcitrant to traditional clinical approaches. One such genetic disease is $\beta$-thalassemia major (Cooley's anemia). This disease is caused by either a point mutation or the deletion of several nucleotides in the $\beta$-globin gene, and it threatens the lives of millions of people in China. In the present study, we successfully generated iPSCs from fibroblasts collected from a 2-year-old patient who was diagnosed with a homozygous $41 / 42$ deletion in his $\beta$-globin gene. More importantly, we successfully corrected this genetic mutation in the $\beta$-thalassemia iPSCs by homologous recombination. Furthermore, transplantation of the genetically corrected iPSCs-derived hematopoietic progenitors into sub-lethally irradiated immune deficient SCID mice showed improved hemoglobin production compared with the uncorrected iPSCs. Moreover, the generation of human $\beta$-globin could be detected in the mice transplanted with corrected iPSCs-derived hematopietic progenitors. Our study provides strong evidence that iPSCs generated from a patient with a genetic disease can be corrected by homologous recombination and that the corrected iPSCs have potential clinical uses.

Keywords: iPS; thalassemia

Cell Research (2012) 22:637-648. doi:10.1038/cr.2012.23; published online 7 February 2012

\section{Introduction}

Induced pluripotent stem cells (iPSCs) can be derived from differentiated somatic cells by simultaneously expressing several transcription factors that are normally expressed at high levels in embryonic stem cells (ESCs) [1-6]. This process allows for the generation of diseasespecific iPSCs that can be used to model human diseases, and ultimately iPSCs may lead to the development of

\footnotetext{
*These two authors contributed equally to this work.

Correspondence: Shaorong Gao ${ }^{\mathrm{a}}, \mathrm{Ze}$ Yang ${ }^{\mathrm{b}}$

${ }^{a}$ Tel: +86-10-80728967; Fax: +86-10-80727535

E-mail: gaoshaorong@nibs.ac.cn

${ }^{\mathrm{b}}$ Tel: +86-10-58115081

E-mail: yangze016@yahoo.com.cn

Received 5 September 2011; revised 6 December 2011; accepted 27 December 2011; published online 7 February 2012
}

cures for many genetic and degenerative diseases [7-10].

$\beta$-thalassemia, one of the most common genetic diseases, is an inherited blood disorder that is characterised by a reduction in the synthesis of hemoglobin (HB) subunit $\beta$ (HB $\beta$ chain). The most common molecular defects are either point mutations or small deletions that affect the transcription, splicing or translation of the $H B B$ gene mRNA. Individuals with $\beta$-thalassemia major (also called Cooley's anemia) have severe anemia and hepatosplenomegaly. Without treatment, affected children fail to thrive and have a shortened life expectancy. More importantly, this genetically inherited disease, which is prevalent throughout the southern part of China, has threatened millions of people's lives for decades, and no effective treatments are available.

The generation of iPSCs from patients has raised hopes for curing blood diseases caused by genetic mutations, and a proof of principle study has shown that a 
humanized sickle cell anemia mouse model could be rescued by transplantation of genetically corrected iPSCsderived hematopoietic stem cells [11]. Recent studies from others and ourselves have clearly shown that iPSCs can be produced from human fetal fibroblasts carrying a $\beta$-thalassemia mutation $[12,13]$. However, $\beta$-thalassemia iPSCs have not yet been genetically corrected and the potential in vivo functionality of $\beta$-thalassemia diseasefree iPSCs has not been evaluated.

In the present study, we aimed to further investigate if the genetic mutation in the $\beta$-thalassemia patient-specific iPSCs can be successfully corrected by homologous recombination. Furthermore, the functionality of the genetically corrected iPSCs was examined through in vitro differentiation and in vivo transplantation. Our results demonstrate that genetically corrected iPSCs-derived hematopoietic progenitors (HPs) could differentiate in vivo and produce human $\beta$-globin in a mouse model. These results have important implications for personalized treatment of $\beta$-thalassemia in the future.

\section{Results}

\section{Establishment of patient-specific iPS cell lines}

In the present study, fibroblasts were obtained and cultured from a skin biopsy of a 2-year-old $\beta-41 / 42$ homozygous patient in which the $\beta$-globin gene showed a TCTT deletion between the $41^{\text {st }}$ and $42^{\text {nd }}$ amino acids. Human OCT3/4, SOX2, and KLF4 genes were introduced into these cells via retroviral transduction. Forty-eight hours after transduction, the cells were split with trypsin at a density of $5 \times 10^{4}$ per $100 \mathrm{~mm}$ dish and cultured in iPSC culture medium. Approximately, 40 human ESClike colonies emerged from each dish, and 16 colonies were picked around day 25 after transduction. These colonies showed compact morphologies indistinguishable from hESCs with distinct edges and high nucleus/ cytoplasm ratio (Supplementary information, Figure S1A). These 16 iPSC lines were further expanded, and all showed a normal karyotype after 20 passages without replicative crisis (data not shown). We named these iPSC lines as piPS1 16 .

To further characterize their pluripotency, four piPSC lines were randomly selected and a Chinese hESC line was used as a positive control (Supplementary information, Figure S2). Quantitative RT-PCR results revealed that both total and endogenous expression levels of the three key factors that we transduced in piPSCs, OCT3/4, $S O X 2$, and KLF4, were similar to hESCs (Supplementary information, Figure S1B). These results indicate that the three exogenous genes were effectively silenced. Quantitative RT-PCR results showed that these piPSC lines expressed other ESC marker genes correctly, which were comparable to hESCs (Supplementary information, Figure S1B). Bisulfite-sequencing analysis confirmed that demethylation occurred in the promoter regions of genes coding for the pluripotency-associated transcription factors OCT3/4 and NANOG in piPSCs (Supplementary information, Figure S3A). Immunofluorescent staining analysis of these piPSC lines revealed the expression of transcription factor markers such as OCT3/4, SOX2, and NANOG, as well as surface markers such as SSEA4, TRA1-60, and TRA1-81, which are characteristic of hESCs (Supplementary information, Figure S1C).

To assess their differentiation ability, we differentiated the piPSC lines both in vitro and in vivo. piPSC-derived embryoid bodies were cultured for 8 days (Supplementary information, Figure S3B). They could readily differentiate into ectoderm, mesoderm, and endoderm derivatives as determined by cell morphology and by immunostain-

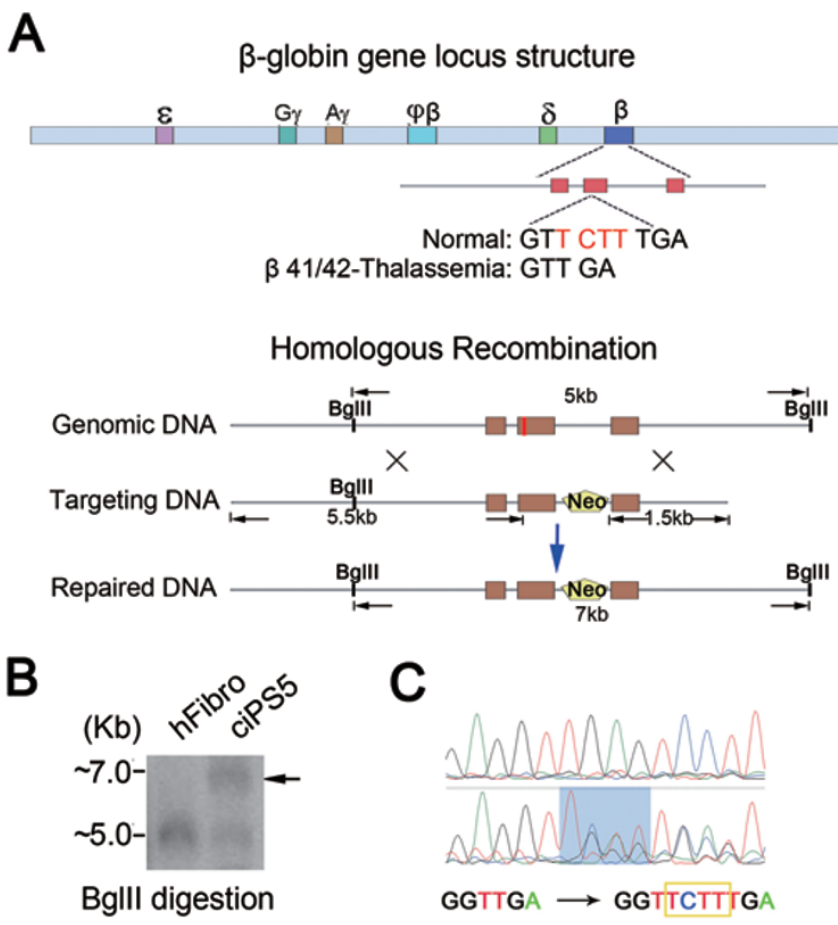

Figure 1 Genetic correction of $\beta-41 / 42$ mutation in piPS cells. (A) Schematic representation of the gene-targeting strategy for correcting the four nucleotides deletion in $\beta$-globin gene in piPS cells. (B) Southern blot analysis of patient fibroblast cells and ciPSCs. Genomic DNA from patient fibroblast cells and ciPS cells were digested by Bglll. The approximate molecular weights (kb) corresponding to the bands are indicated. Arrowhead indicates the integration of targeting vector. (C) Sequencing results of the $\beta-41 / 42$ mutation site of $H B B$ gene in both piPSCs (upper panel) and ciPSCs (lower panel). The sequence changes are indicated at the bottom. 
A

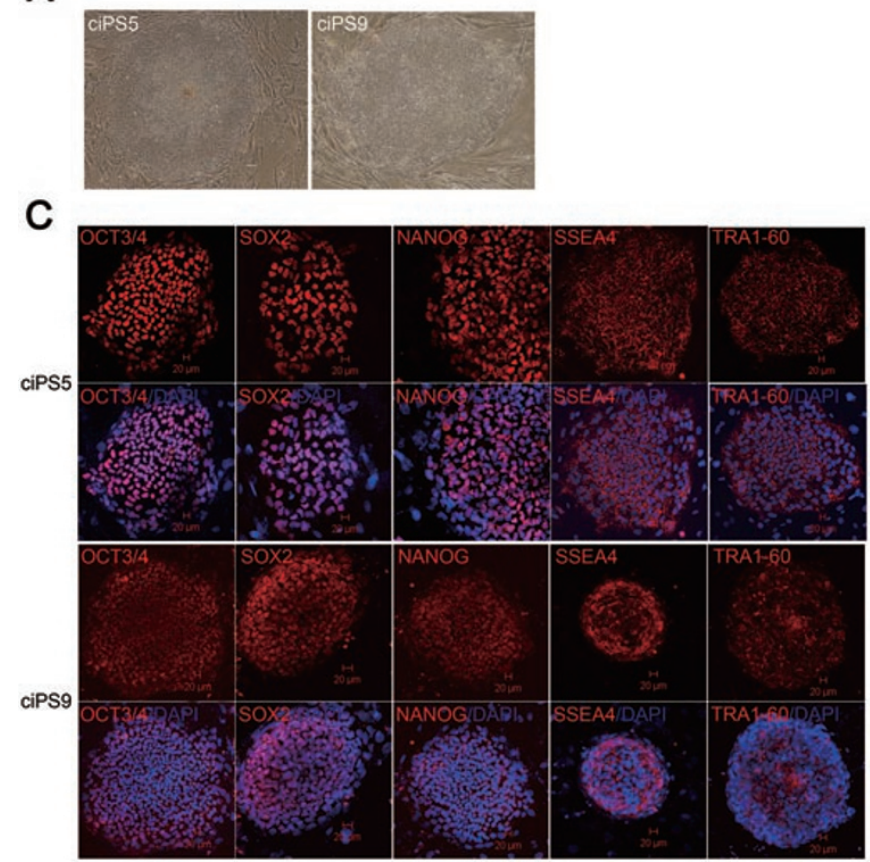

B

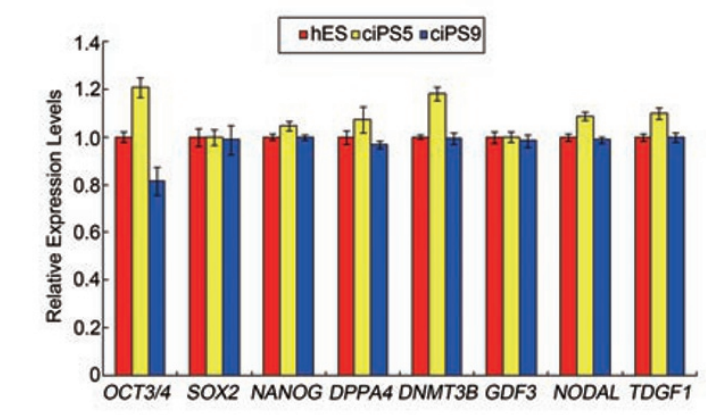

D

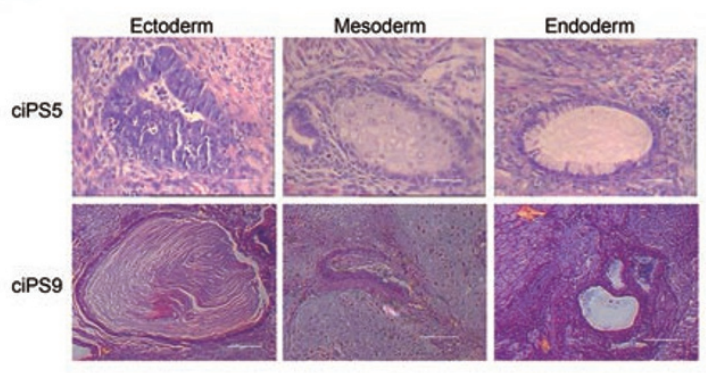

Figure 2 Characterization of genetic corrected iPS cells. (A) Morphologies of two ciPSC lines; scale bar, $100 \mu \mathrm{m}$. (B) The expression levels by qRT-PCR of ESC marker genes in hES cells, ciPS5 cells and ciPS9 cells. Transcript levels were normalized to GAPDH. Error bars represent SD $(n=3)$. (C) Immunostaining of ESC markers in ciPS5 and ciPS9 cells; scale bar, 20 $\mu \mathrm{m}$. (D) H\&E staining of teratomas containing tissues of all three germ layers derived from ciPS5 and ciPS9 cells; scale bar, $20 \mu \mathrm{m}$.

ing with specific antibodies against SMA, Troponin I, Vimentin, and PDX1 (Supplementary information, Figure $\mathrm{S} 3 \mathrm{C})$. Directed in vitro differentiation results showed that these iPSCs could give rise to hematopoietic progenitor cells (see below). We also injected the four piPSC lines into SCID mice subcutaneously for teratoma formation, which is the most stringent test available for assessing the pluripotency of hESCs. In all cases, teratomas were formed 8 weeks after injection. They contained derivatives from all three embryonic germ layers, including columnar epithelium (endoderm), muscle, cartilage (mesoderm), cuticular epithelium, and neural rosette (ectoderm) (Supplementary information, Figure S1D). In summary, we successfully generated $16 \beta$-thalassemia patient-specific iPSC lines. Furthermore, there were no conspicuous differences between these iPSCs and hESCs in their ability to either self-renew or differentiate.

\section{Genetic correction of $\beta-41 / 42$ mutation in piPS cells}

Next, we tried to correct the genetic mutation in the piPSCs by specific gene targeting. We used the classic homologous recombination method following established protocols [14]. The targeting scheme was summarized in
Figure 1A. The targeting plasmid was constructed as previously reported [15]. After linearization, the vector was introduced into piPS7 cells by electroporation, followed by G418 selection. Drug-resistant colonies emerged 2 weeks after electroporation, which were picked and expanded in iPSC culture medium. They were then further verified by PCR screen for Neo fragment integration into the genomic DNA (data not shown), Southern blot analysis (Figure 1B), and direct sequencing of the $H B B$ gene (Figure 1C). The targeting efficiency was summarized in Supplementary information, Table S1. In the targeting process, we used $10^{7}$ piPSCs in each of the three independent electroporation experiments, and finally obtained two successfully corrected iPSC lines with morphologies similar to hESCs (Figure 2A). Both of the corrected iPSC lines showed a normal karyotype without replicative crisis after more than 30 passages (data not shown). We named these iPSCs as ciPSCs.

For pluripotency characterization of the ciPSCs, the same procedures mentioned above were adopted. Quantitative RT-PCR results revealed that ES marker genes are expressed in the ciPSCs (Figure 2B). Immunofluorescence analysis showed that $\mathrm{hESC}$ transcriptional markers 
(OCT3/4, SOX2, NANOG) and surface markers (SSEA4, TRA1-60) were all expressed in the ciPSCs (Figure 2C). Teratomas consisting of derivatives from all three embryonic germ layers also formed after the subcutaneous injection of the two cell lines into SCID mice (Figure 2D). Overall, the process of generating disease-corrected iPSCs by targeted homologous recombination did not compromise their ability to either self-renew or differentiate, which is a prerequisite for further hematopoietic applications.

To avoid cell line contaminations, STR analyses were performed in all iPSC lines we derived (16 piPSC lines and 2 ciPSC lines) as well as the patient fibroblast cells. All 19 cell lines showed the same origin with identical signal peaks, suggesting that all the iPSCs were derived from the patient fibroblast cells, with no contamination (Figure 3).
Global gene-expression profile analysis of iPSCs

Before applying the genetically corrected iPSCs for hematopoietic differentiation, we compared the global gene-expression profiles of hESCs, piPS7 cells, ciPS5 cells and patient fibroblast cells using DNA microarrays (GSE30220). Pearson correlation analysis revealed that piPS7 cells and ciPS5 cells are clustered very closely with hESCs but separately from fibroblasts (Figure 4A). Scatter plots of the global gene expression profiles obtained from cDNA microarrays demonstrated that both patient-specific iPSCs and targeted iPSCs exhibit a distribution pattern of gene expression that is almost identical to each other but completely different from fibroblasts (Figure 4B). The data from chip analysis also revealed that the expression levels of ES-marker genes and pluripotency-associated genes in the two iPSC lines were both high, similar to those in hESCs (Figure 4C). These data
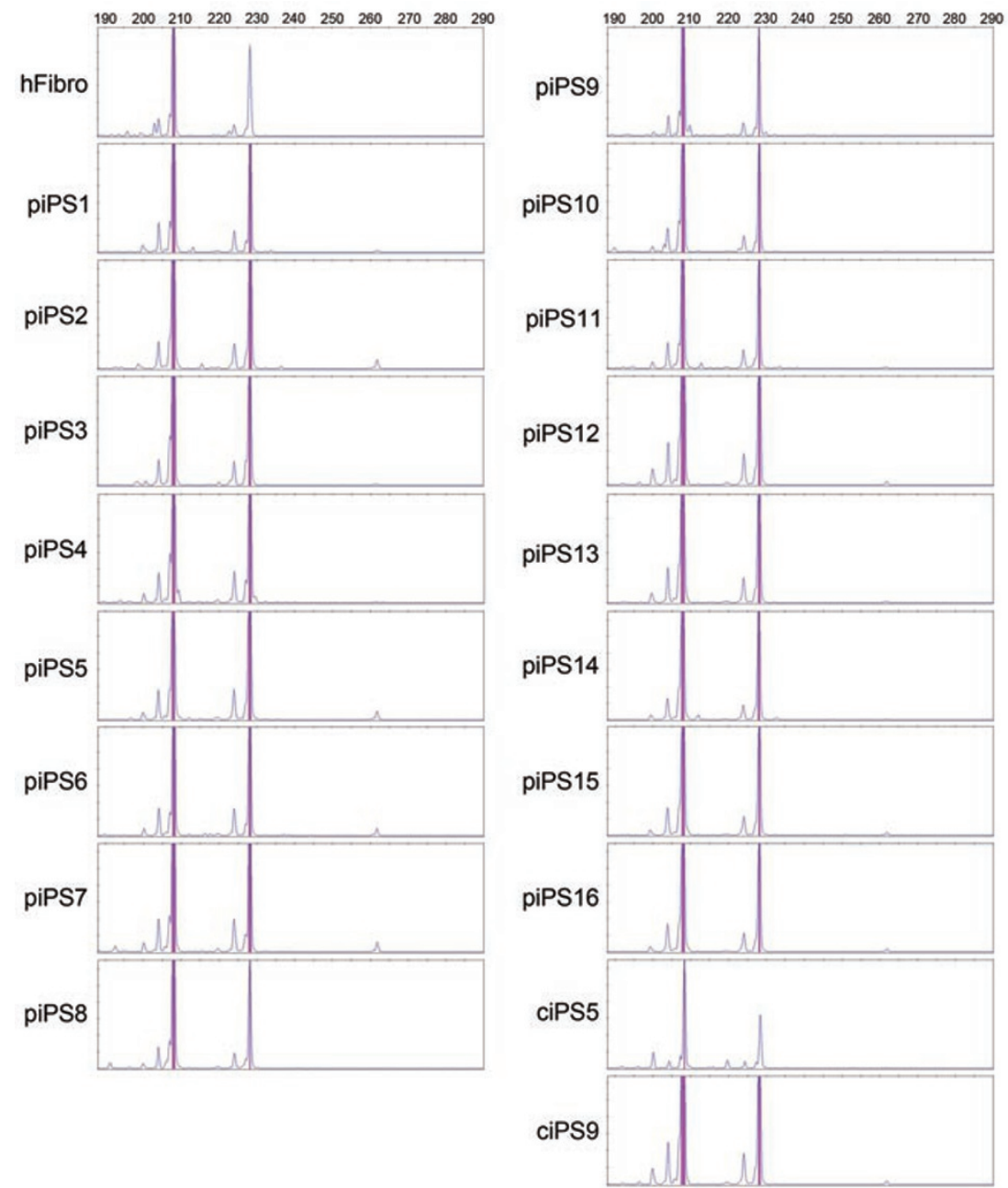

Figure 3 STR analysis of all iPSC lines and patient fibroblast cells. STR D6S2427 was analyzed to confirm all iPSC lines were with the same origin as patient fibroblast cells. 


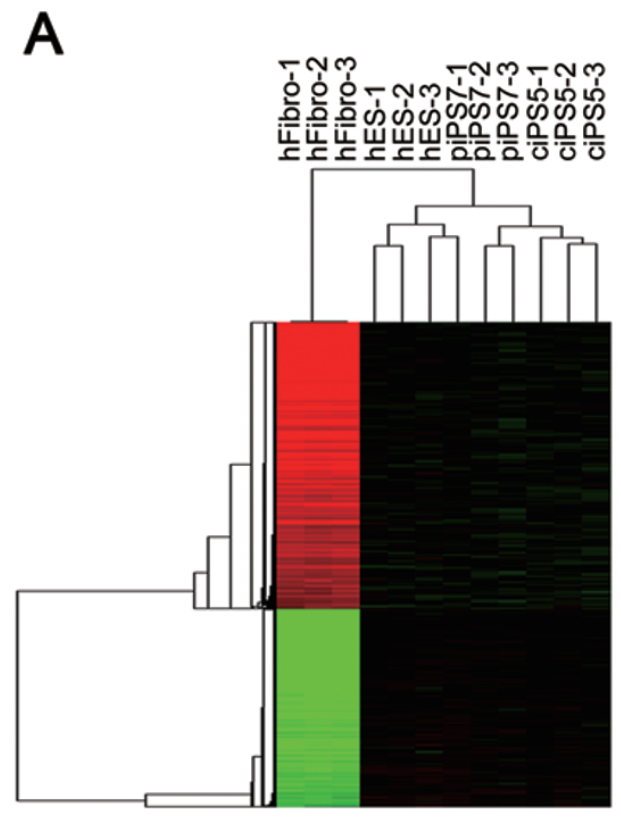

B

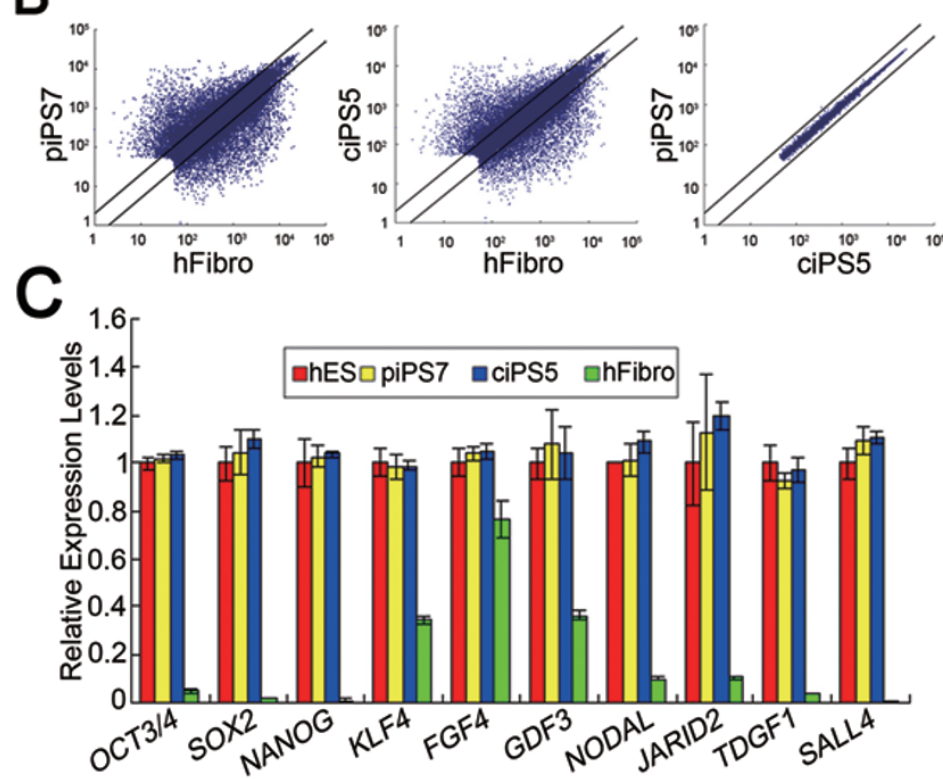

Figure 4 Global gene expression analysis by microarrays. (A) Pearson correlation analysis of probes was performed to cluster hESC, piPS7 cells, ciPS5 cells, and patient fibroblast cells. Red indicates increased expression compared with median levels of the four samples, whereas green means decreased expression. (B) Scatter plots of the global gene expression patterns comparing piPS7 cells with fibroblasts, ciPS5 cells with fibroblasts, and piPS7 cells with ciPS5 cells (from left to right). Black lines indicate two-fold changes in gene expression levels. (C) Expression levels of ES-specific and pluripotency-associated genes in hES cells, piPS7 cells, ciPS5 cells and fibroblast cells. Error bars represent SD $(n=3)$.

above demonstrated that the iPSCs we derived showed an almost identical gene expression pattern to hESCs. Moreover, gene targeting by homologous recombination did not affect ESC-like properties of iPSCs.

\section{Improved functionality of HPs derived from genetically corrected iPSCs}

To examine the hematopoietic differentiation ability and to compare the erythroid differentiation ability of the piPSCs and ciPSCs, four piPSC lines and two ciPSC lines characterized above, as well as hESCs were co-cultured with OP9 stromal cells separately using protocols established previously [16]. Eight days after co-culture, hematopoietic cell markers including CD43, CD235a, and CD45 were analyzed by flow cytometry. The timing and ratios of the appearance of HPs appeared similar in all seven cell lines. Flow cytometry analysis showed that the proportion of $\mathrm{HP}\left(\mathrm{CD} 43^{+} \mathrm{CD} 31^{+}\right)$derived from the four piPSC lines was around $3.3 \%$, which was comparable to those obtained in the two ciPSC lines (around 3.6\%) and the hESCs (3.88\%) (Supplementary information, Figure S4). These results indicate that both piPS cells and ciPS cells could undergo successful hematopoietic differentiation in vitro. We further improved the OP9 coculture system by adding hematopoietic cytokines such as SCF, IL-3, IL-6, and Flt3L into the culture medium, and the results showed that the proportions of $\mathrm{CD} 43^{+}$ cells were doubled in all seven cell lines after 8 days of co-culture in the presence of cytokines (Supplementary information, Figure S5). The derived erythro-megakaryocytic progenitors $\left(\mathrm{CD} 43^{+} \mathrm{CD} 235 \mathrm{a}^{+} \mathrm{CD} 45^{-}\right)$were found comparable among the seven cell lines tested (Supplementary information, Figure S5).

To further assess the hematopoietic differentiation capability of these cells, we performed clonogenic progenitor assays with the piPSC- and ciPSC- as well as hESC-derived CD $34^{+}$cells after MACS (Supplementary information, Figure S6). After seeding the same amounts of cells in MethoCult medium, CFU-Ms and CFU-Gs firstly appeared on day 3 in all cell lines, whereas CFU-E first appeared on day 8 . We examined the morphologies and amounts of CFUs of different lineages derived from respective cell lines around day 14, and found that both morphologies and proportions of different lineages of CFUs appeared similar in all cell lines after hematopoietic differentiation induction (Figure 5A and 5B).

We focused on examining the morphologies of CFUEs derived from the three types of cells, piPSCs, ciPSCs, and hESCs. Although similar in size, the CFU-Es derived from ciPSCs appeared a little more hypochromic than 
A

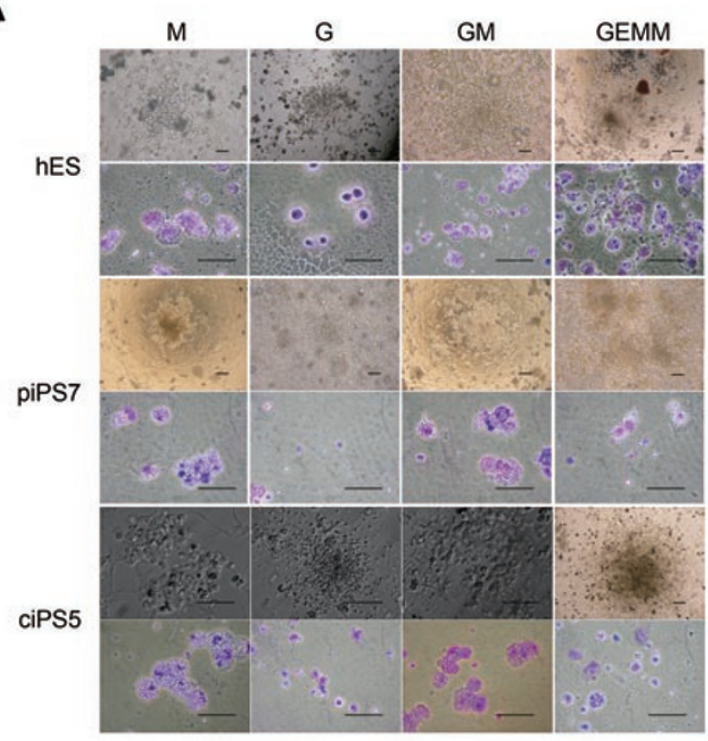

B

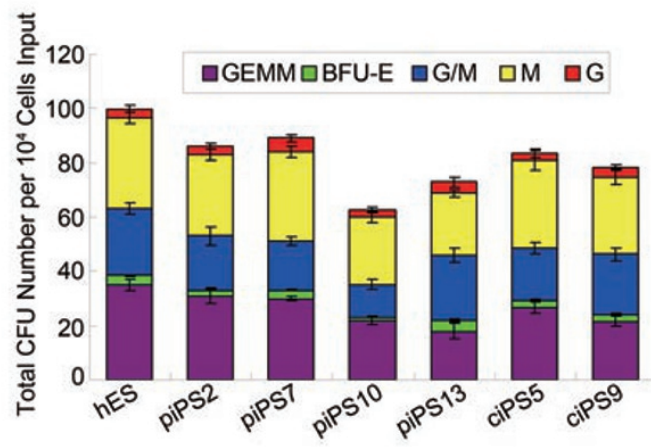

C

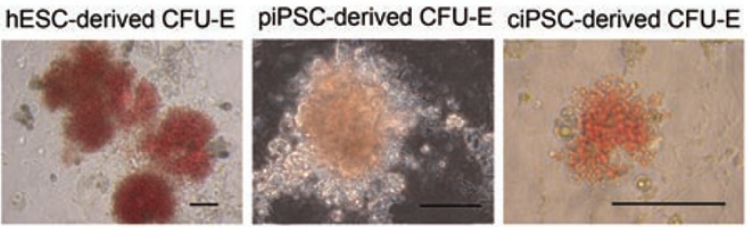

Figure 5 CFU assays performed on $\mathrm{CD}_{3} 4^{+}$cells derived from hES-HPs, piPS-HPs, and ciPS-HPs. (A) Morphology of hematopoietic CFU subtypes derived from hES-HPs, piPS-HPs, and ciPS-HPs. Scale bar $50 \mu \mathrm{m}$. (B) Distribution of colony subtypes in clonogenic progenitor assays on day 14 differentiated from hES-HPs, piPS-HPs, and ciPS-HPs. (C) Morphologies of CFU-Es derived from hES-HPs, piPS-HPs, and ciPS-HPs. Scale bar $50 \mu \mathrm{m}$. M, macrophage; G, granulocyte; GM, multipotent colonies containing granulocyte and macrophage elements (CFU-GM); GEMM, multipotent colonies containing granulocyte, erythroid, macrophage, and megakaryocyte elements (CFUGEMM); HP, hematopoietic progenitor cell.

those derived from hESCs, while they were much more reddish than those derived from piPSCs (Figure 5C). Overall, similar to previous reports [16], no significant differences were observed among the pluripotent cell lines after hematopoietic differentiation in vitro.

Next, in vivo hematopoietic differentiation assay was performed to examine the in vivo function. Female SCID mice with similar weight were sub-lethally irradiated by Co-60, and randomly divided into four groups: $\alpha$-MEM group, piPS-HP group, ciPS-HP group, and hES-HP group. Meanwhile, $5 \times 10^{5} \mathrm{CD} 34^{+} \mathrm{HPs}$ derived from piPSCs, ciPSC, and hESCs were enriched by MACS and transplanted into the intra-tibiae of the SCID mice. Six weeks after transplantation, peripheral blood, bone marrow (BM) cells from the transplantation and the nontransplantation sides, or spleen cells were collected and analyzed by flow cytometry using specific human HLA$\mathrm{ABC}$ antibody and human CD71 antibody, separately. Human HLA-ABC ${ }^{+}$cells could be detected at similar ratios in mouse peripheral blood and BM cells (Figure $6 \mathrm{~A}$ ), while human early erythroblasts could be detected at similar ratios in mouse BM cells and spleen cells in all groups, except the control group injected with $\alpha$-MEM (Figure 6B). These results indicate that the transplanted cells were functional and capable of undergoing hematopoiesis in these SCID mice. The presence of the human SRY gene was also detected in genomic DNA extracted from the peripheral blood, BM cells from the transplantation and the non-transplantation sides of the cell-transplanted female SCID mice except the control group (Supplementary information, Figure S7), further confirming that the transplanted human cells performed hematopoiesis in these SCID mice. These results strongly suggest that the transplanted cells derived from the three cell lines were functional in hematopoiesis in these SCID mice, which is consistent with the in vitro studies.

To test whether HPs derived from gene-targeted iPSCs could produce functional $\beta$-globin chains in vivo, red blood cells (RBCs) were collected from the peripheral blood of mice 6 weeks after transplantation, lysed and subjected to HPLC C4 column analysis for measurement of globin chain synthesis. Different from the results of the $\alpha$-MEM group and piPS-HP group, a new small protein absorption peak was observed in the hemolysates of both hES-HP group and ciPS-HP group, which accounted for a similar proportion compared with the expression levels of mouse $\beta$-globin ( $7.34 \%$ and $8.73 \%$ respectively, Figure 6C). Western blot analysis showed that the new protein peak eluted from HPLC reacted with a specific antibody against human $\beta$-globin (Figure 6D). To check whether this protein peak contains other globins such as $\gamma$-globin, we also used a specific human $\gamma$-globin antibody to examine if this protein peak contains $\gamma$-globin. Western blot results showed that no human $\gamma$-globin could be detected in this protein peak (data not shown). 
A

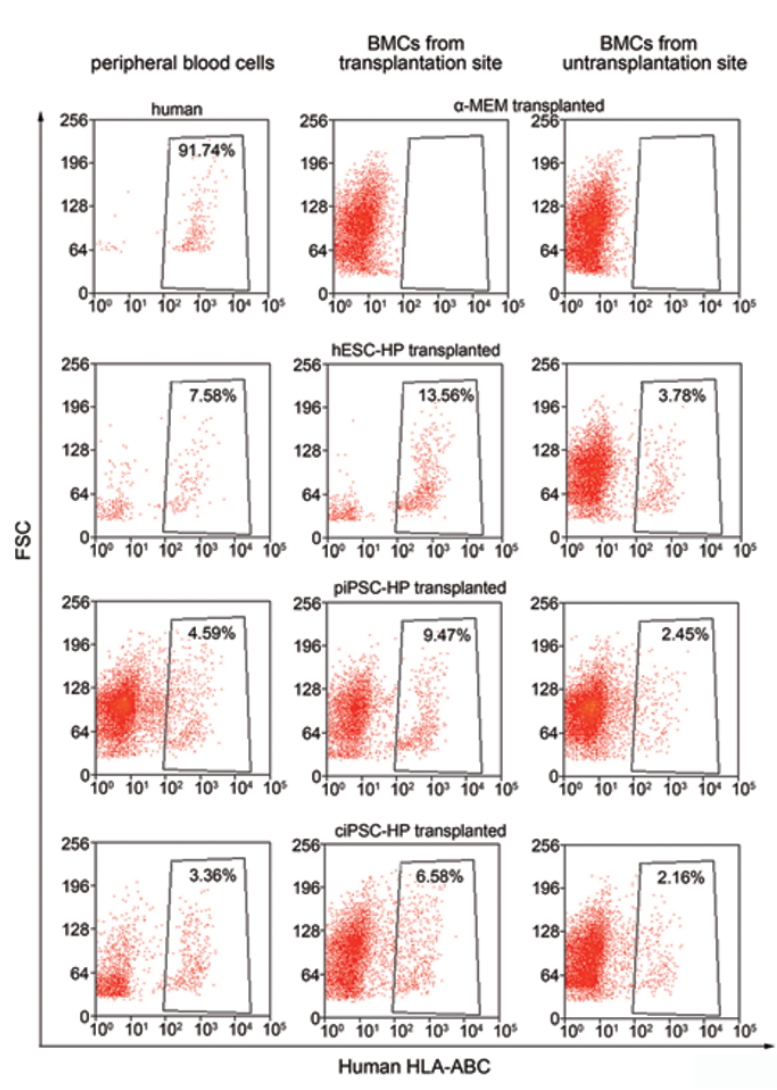

B

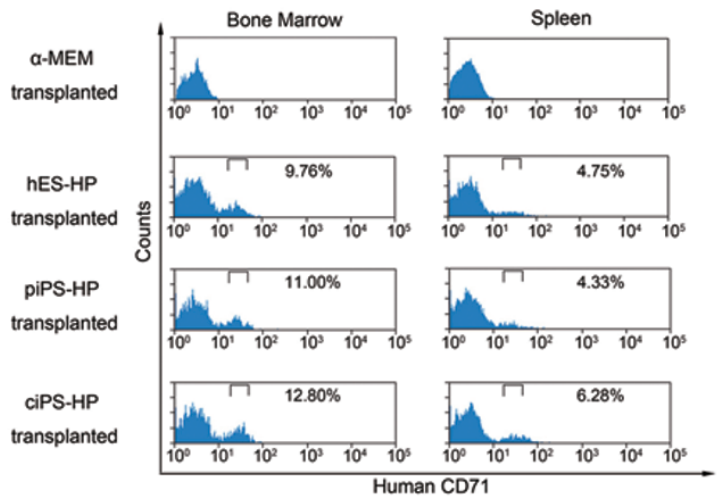

C

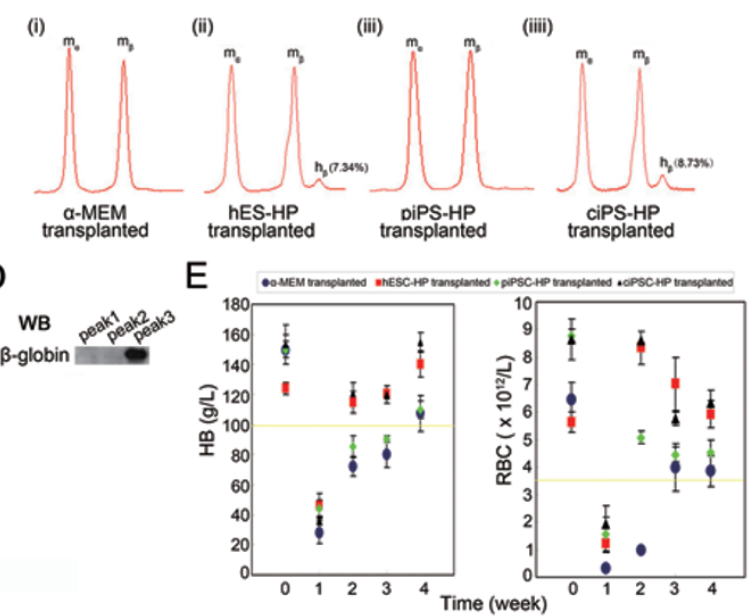

Figure 6 Hematopoietic differentiation of $\beta$-thalassemia-iPS cells and genetic corrected iPS cells in vivo. (A) Flow cytometry analysis of human cell (human $\mathrm{HLA}-\mathrm{ABC}^{+}$) ratio in peripheral blood cells, BM cells from transplantation sides and untransplantation sides of the four groups of SCID mice transplanted with $\alpha$-MEM, hES-HPs, piPS-HPs, and ciPS-HPs separately 6 weeks after transplantation. (B) Flow cytometry analysis of human $\mathrm{CD} 71^{+}$cells ratio in BM cells and spleen cells of the four group SCID mice transplanted with $\alpha$-MEM, hES-HPs, piPS-HPs, and ciPS-HPs, separately 6 weeks after transplantation. (C) HPLC analysis of human $\beta$-globin gene expression in peripheral blood cells of the four groups of SCID mice transplanted with $\alpha$-MEM (i), hES-HPs (ii), piPS-HPs (iii), and ciPS-HPs (iiii) separately 6 weeks after transplantation. (D) Western blotting of three protein absorption peaks eluted from HPLC using hemolysates from ciPS-HP group mice. Specific human $\beta$-globin antibody was used. (E) Changes in HB levels (left panel) and RBC accounts (right panel) in the peripheral blood of the four groups of SCID mice transplanted with $\alpha$-MEM, hES-HPs, piPS-HPs, and ciPS-HPs separately from week 0 to week 4 after transplantation. Error bars represent S.D. $(n=5)$. HB, hemoglobin; RBC, red blood cell; BM, bone marrow; HP, hematopoietic progenitor cell.

Peripheral blood from all four groups of transplanted mice was also collected separately for human $\beta$-globin and $\gamma$-globin expression analyses by qRT-PCR and western blot. Real-time PCR results showed that while the expression level of human $\gamma$-globin was similar among the hESC-HP, piPSC-HP, and ciPSC-HP groups, the expression level of human $\beta$-globin was extremely low in peripheral blood from the piPSC-HP group. We also found that the ciPSC-HP group expressed about halflevel of human $\beta$-globin as compared with the hESC-HP group, which might be due to the fact that only one allele was corrected during the targeting process (Figure 7A).
For western blot analysis, we could not detect human $\beta$-globin expression in the peripheral blood of both the $\alpha$-MEM group and piPSC-HP group. The expression level of human $\beta$-globin was lower in the ciPSC-HP group than in the hESC-HP group. Human $\gamma$-globin expression could be detected in all groups except the $\alpha$-MEM group (Figure 7B). These data strongly suggest that the genetargeted iPSCs, as well as hESCs, could produce adult $\beta$-globin after hematopoietic differentiation in vivo.

Finally, peripheral blood cells collected from the transplanted mice were analyzed. Blood count follow-up tests were performed up to 4 weeks after hematopoietic 
A
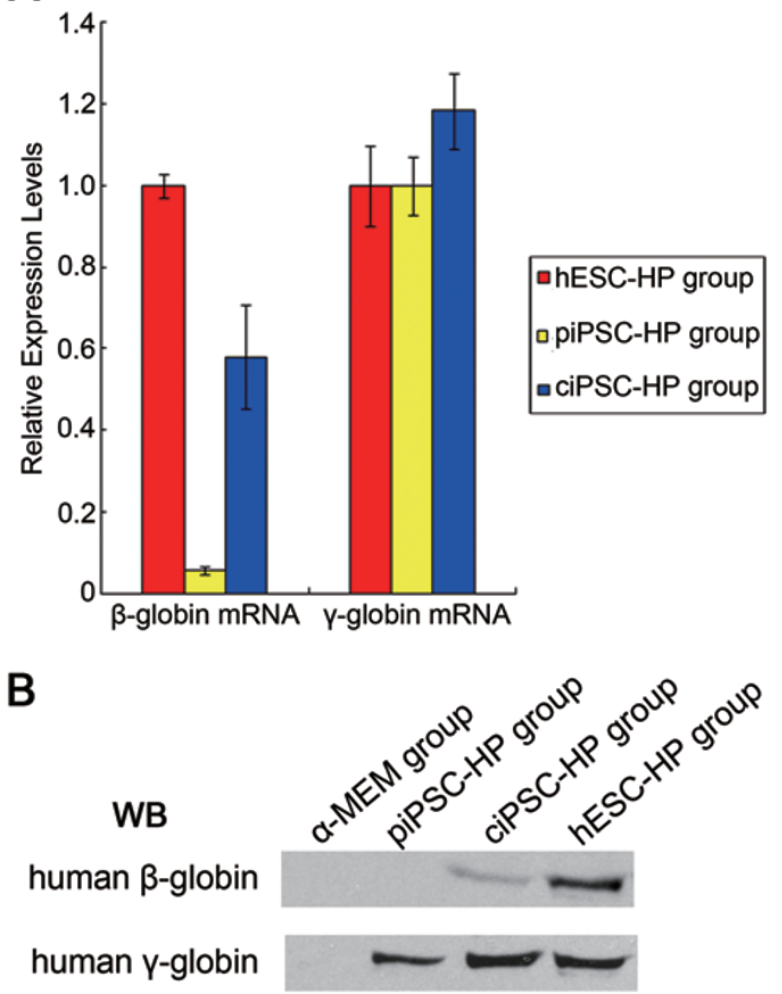

Figure 7 Analysis of human $\beta$-globin and $\gamma$-globin expression in the peripheral blood collected from all groups of transplanted mice. (A) qRT-PCR analysis of human $\beta$-globin and $\gamma$-globin mRNA expression in the peripheral blood collected from hESCHP, piPSC-HP, and ciPSC-HP groups. (B) Western blot analysis of human $\beta$-globin and $\gamma$-globin expression using specific antibodies in the peripheral blood collected from all groups of transplanted mice.

transplantation (Table 1). Due to irradiation, both levels of $\mathrm{HB}$ and RBCs in the peripheral blood dropped greatly under the base line (which is $100 \mathrm{~g} / 1$ and $3.6 \times 10^{12} / 1$, respectively) in all four groups of transplanted mice one week after transplantation. The HB levels in the hES-HP and ciPS-HP-transplanted mice elevated above $100 \mathrm{~g} / 1$ from the second week after transplantation. In contrast, the HB levels in the rest two groups of mice increased slowly and remained below the normal base line until the $4^{\text {th }}$ week after transplantation (Figure 6E, Table 1), from which time point, all four groups exhibited normal HB levels. These blood routine data suggest that genetically corrected iPSCs-derived HP can positively stimulate hematopoiesis in these SCID mice after irradiation. Thus, our results collectively demonstrate that the genetically corrected iPSCs, like human ESCs, have the ability to give rise to hematopoietic progenitors that undergo normal hematopoiesis in a sub-lethally SCID mouse model.

\section{Discussion}

In this study, we successfully derived 16 patientspecific iPS cell lines from a 2-year-old boy suffering from $\beta$-thalassemia major. Furthermore, we corrected the genetic mutation of the disease by homologous recombination using a classic gene-targeting approach. By transplantation into an immune-deficient mouse model, we demonstrated that the production of HB could be significantly increased when disease-corrected iPSCs-derived HPs were transplanted and moreover, the production of human-specific $\beta$-globin could be detected. Our study advances the field by demonstrating a potential use of the iPSC technology in clinical treatment of $\beta$-thalassemia major.

Correction of the genetic mutation in iPS cells derived from the individual patient with genetic disease is the most critical step towards personalized regenerative medicine practice. Although two corrected colonies were finally obtained using the traditional homologous recombination approach, the targeting efficiency is very low and the targeting method needs to be further optimized. Recently, introduction of a transgene into the iPS cells or correction of the genetic mutation by zinc finger nuclease- or bacteria artificial chromosome (BAC)-based methods offers alternative ways to obtain genetically corrected iPS cells [17-19]. More recently, helper-dependent adenoviral vectors were used to provide a more efficient method for correcting mutations in human pluripotent stem cells $[20,21]$. It is desirable to further improve the efficiency of correcting the $\beta$-globin gene mutation in the iPS cells produced in the present study by comparing the different approaches listed above.

Before the clinical application of iPSCs can finally be realized, a number of hurdles must be overcome. The recent generation of protein, mRNA, and microRNA reprogrammed iPS cells provides a more ideal approach to obtaining safer patient-specific iPS cells [22-25]. Subsequently, selecting good quality iPS cells will be needed for further genetic manipulation. One major possibility causing incomplete erasing of the epigenetic memory inherited from the somatic cells and the immunogenicity of iPS cells as observed recently might be due to incomplete reprogramming of the somatic genome [26-29]. Finally, the strategy of directed differentiation of corrected iPS cells into specific cell types has to be optimized in the future. Recent studies have shown that the direct conversion of one cell type into another is feasible both in vivo and in vitro [30-34]. It remains unknown, however, if these trans-differentiated cells are functional, because the length of the telomeres in these cells may not be properly restored. Interestingly, telomere length can indeed 
Table 1 Summary of blood routine parameters of four groups of SCID mice transplanted with $\alpha$-MEM, hES-HP, piPS-HP, and ciPS-HP from week 0 to week 4 after irradiation

\begin{tabular}{|c|c|c|c|c|c|}
\hline & Weight (g) & $\mathrm{RBC}\left(\times 10^{12} / 1\right)$ & HB (g/l) & $\operatorname{MCV}(f l)$ & $\mathrm{MCH}(\mathrm{pg})$ \\
\hline \multicolumn{6}{|l|}{0 Week } \\
\hline$\alpha$-MEM group & $17.67 \pm 0.53$ & $6.45 \pm 1.23$ & $150.00 \pm 5.36$ & $46.20 \pm 3.25$ & $37.50 \pm 3.86$ \\
\hline piPSC-HP group & $17.68 \pm 0.47$ & $8.78 \pm 0.23$ & $150.00 \pm 9.81$ & $42.80 \pm 0.79$ & $17.30 \pm 0.86$ \\
\hline ciPSC-HP group & $17.14 \pm 0.30$ & $8.64 \pm 0.73$ & $153.23 \pm 13.25$ & $43.20 \pm 0.38$ & $18.90 \pm 2.30$ \\
\hline hESC-HP group & $13.15 \pm 0.19$ & $1.24 \pm 0.31$ & $46.00 \pm 8.48$ & $41.90 \pm 1.69$ & $55.50 \pm 8.06$ \\
\hline piPSC-HP group & $13.34 \pm 0.56$ & $1.56 \pm 0.64$ & $44.00 \pm 5.66$ & $44.80 \pm 0.85$ & $32.30 \pm 5.73$ \\
\hline ciPSC-HP group & $14.11 \pm 0.36$ & $1.94 \pm 0.64$ & $36.00 \pm 2.83^{*}$ & $38.70 \pm 6.36$ & $113.10 \pm 5.87$ \\
\hline \multicolumn{6}{|l|}{2 Week } \\
\hline ciPSC-HP group & $14.76 \pm 0.45$ & $8.59 \pm 0.33$ & $120.5 \pm 7.78$ & $42.85 \pm 2.33$ & $13.95 \pm 0.35$ \\
\hline \multicolumn{6}{|l|}{3 Week } \\
\hline$\alpha$-MEM group & $16.05 \pm 0.78$ & $3.98 \pm 1.87$ & $80.50 \pm 9.19 * *$ & $43.05 \pm 0.07$ & $18.76 \pm 9.09$ \\
\hline hESC-HP group & $15.79 \pm 0.63$ & $7.02 \pm 1.98$ & $120.50 \pm 23.30$ & $45.50 \pm 0.57$ & $19.45 \pm 1.34$ \\
\hline piPSC-HP group & $16.52 \pm 0.60$ & $4.46 \pm 0.28$ & $90.50 \pm 2.12 * *$ & $43.6 \pm 0.85$ & $13.35 \pm 3.46$ \\
\hline ciPSC-HP group & $16.30 \pm 0.31$ & $5.75 \pm 0.24$ & $120.00 \pm 25.46$ & $44.25 \pm 0.92$ & $18.95 \pm 4.03$ \\
\hline \multicolumn{6}{|l|}{4 Week } \\
\hline$\alpha$-MEM group & $17.11 \pm 0.25$ & $3.85 \pm 1.54$ & $107.50 \pm 12.02 * *$ & $44.30 \pm 0.57$ & $136.3 \pm 23.05$ \\
\hline hESC-HP group & $17.01 \pm 0.23$ & $5.93 \pm 0.48$ & $140.00 \pm 12.73$ & $46.35 \pm 2.47$ & $34.25 \pm 0.78$ \\
\hline
\end{tabular}

Values represent the mean \pm SD. Statistical significance was determined for HB of $\alpha$-MEM, piPS-HP, and ciPS-HP groups compared with that of hES-HP group; $P$ values were calculated using Student's $t$ test; $n=5$ each group. ${ }^{*} P<0.01$; $* * P<0.05$. Weight values were taken from age-matched mouse groups (6 weeks of age). RBC indicates red blood cell count; HB, hemoglobin; MCV, mean corpuscular volume; $\mathrm{MCH}$, mean corpuscular hemoglobin; fl, femoliter.

be restored in iPSCs $[35,36]$. In conclusion, our study provides strong evidence indicating that the iPSC technology combined with gene targeting could potentially be used to cure genetic diseases such as $\beta$-thalassemia major.

\section{Materials and Methods}

\section{Cell culture}

Patient's skin fibroblasts and 293T cells were maintained in Dulbecco's modified medium (DMEM, Invitrogen) supplemented with $10 \%$ fetal bovine serum (FBS, Hyclone). Human ES cells were maintained on irradiated MEF feeder cells in human ES cell medium: Knockout DMEM (Invitrogen) supplemented with 15\% knockout serum replacement (Invitrogen), 5\% inactivated FBS (Hyclone), $4 \mathrm{ng} / \mathrm{ml}$ bFGF (Peprotech), $10^{-4} \mathrm{M}$ non-essential amino acids (Millipore), $10^{-4} \mathrm{M} \beta$-mercaptoethanol (Millipore), $2 \mathrm{mM} \mathrm{L}$ glutamax (Invitrogen) and $50 \mu \mathrm{g} / \mathrm{ml}$ penicillin/streptomycin (Millipore). iPS cells were maintained on irradiated MEF feeder cells in DMEM/F12 (Invitrogen) supplemented with 20\% knockout serum replacement (Invitrogen), $10 \mathrm{ng} / \mathrm{ml}$ bFGF (Peprotech), $10^{-4} \mathrm{M}$ non-essential amino acids (Millipore), $10^{-4} \mathrm{M} \beta$-mercaptoethanol (Millipore), $2 \mathrm{mM} \mathrm{L}$-glutamax (Invitrogen) and $50 \mu \mathrm{g} / \mathrm{ml}$ penicillin/streptomycin (Millipore).

\section{Retroviral production and patient-specific iPS cell genera-} tion

The plasmids pMIG containing human OCT4, SOX2, and $K L F 4$, obtained from Addgene, were transiently co-transfected with package plasmids into 293T cells using the transfect reagent Vigofect (Vigorous). Viral supernatants were harvested after $48 \mathrm{~h}$, filtered through a $0.45 \mu \mathrm{m}$ low protein binding cellulose acetate filter (Millipore), and concentrated by centrifugation. A total of $5 \times$ 
$10^{4}$ patient fibroblast cells were incubated with virus for $24 \mathrm{~h}$ and then seeded onto irradiated MEF feed cells after 5 days. iPS cell culture medium was substituted after 7 days. iPSC colonies were manually picked and mechanically dissociated for following passage.

\section{Karyotype analysis}

The iPS cells were first incubated in culture medium containing $0.25 \mu \mathrm{g} / \mathrm{ml}$ colcemid (Invitrogen) for $4 \mathrm{~h}$ and then the cells were harvested and incubated in $0.4 \%$ sodium citrate, $0.4 \%$ chloratum Kaliumat $(1: 1, \mathrm{v} / \mathrm{v})$ at $37{ }^{\circ} \mathrm{C}$ for $5 \mathrm{~min}$ and fixed in a methanol:acetic acid mixture $(3: 1, \mathrm{v} / \mathrm{v})$ three times. After Giemsa staining, at least 20 chromosome karyoschisis images were examined.

\section{qRT-PCR analysis}

Total RNA was extracted from cells using TRIzol reagent (Invitrogen) according to the manufacturer's instructions. Complementary DNA synthesis was performed with the M-MLV Reverse Transcriptase Kit (Promega) following the manufacturer's instructions. Quantitative RT-PCR was performed using a SYBR Greenbased PCR Master Mix (Takara) and signals were detected with ABI7500 Real-Time PCR System (Applied BioSystems).

\section{Alkaline phosphatase staining and immunocytochemistry}

Alkaline phosphatase staining was performed using the ALKALINE PHOSPHATASE SUBSTRATE Kit (Sigma) according to the manufacturer's instructions. For immunocytochemistry, cells were fixed with PBS containing 4\% paraformaldehyde for $15 \mathrm{~min}$ at room temperature. After being washed with TBST, the cells were treated with PBS containing $0.1 \%$ Triton X-100 for $15 \mathrm{~min}$ at room temperature and then incubated with PBS containing $4 \%$ normal goat serum (Millipore) for $30 \mathrm{~min}$ at room temperature. The following primary antibodies were used in this study: SSEA4 (1:50, ES Cell Marker Sample Kit, Millipore), TRA1-60 (1:50, ES Cell Marker Sample Kit, Millipore), TRA-1-81 (1:50, ES Cell Marker Sample Kit, Millipore), SOX2 (1:500, Abcam), NANOG (1:500, Abcam), and OCT4 (1:50, ES Cell Marker Sample Kit, Millipore), PDX-1 (1:200, Millipore), Troponin (1:200, Millipore), SMA (1:200, Millipore), Vimentin ((1:200, Millipore), and Pan Cytokeratin ((1:200, Millipore). The following secondary antibodies were used in this study: Alexa 594-conjugated goat anti-rabbit $\operatorname{IgG}(1: 500$, Invitrogen), Alexa 594-conjugated goat anti-mouse IgM (1:500, Invitrogen) and Alexa 594-conjugated goat antimouse IgG (1:500, Invitrogen). Nuclei were stained with DAPI. Stained cells mounted on slides were observed on an LSM 510 META confocal microscope (Zeiss).

\section{In vitro differentiation}

To generate the embryoid bodies, iPS cells were harvested by mechanical dissociation. The clumps of cells were transferred to a 6-well ultralow attachment plates (Costa, Corning) in IMDM (Invitrogen) containing 15\% FBS (HyClone), $10^{-4} \mathrm{M}$ non-essential amino acids (Millipore), $10^{-4} \mathrm{M} \beta$-mercaptoethanol (Millipore), 2 $\mathrm{mM}$ L-glutamax (Invitrogen) and $50 \mu \mathrm{g} / \mathrm{ml}$ penicillin/streptomycin (Millipore). The medium was changed every other day. After 8 days, EBs were transferred to gelatin-coated plates and cultured in the same medium for another 8 days.

\section{Teratoma formation}

The iPS cells were harvested by mechanical dissociation, collected into tubes and centrifuged. The pellets were resuspended in DMEM/F12 (Invitrogen). Cells from a confluent 35-mm dish were injected subcutaneously into the groin of a SCID mouse. Eight weeks after injection, the tumors were dissected and fixed with PBS containing 10\% paraformaldehyde (Sigma). Paraffin-embedded tissue was sliced and stained with hematoxylin and eosin.

\section{Plasmid construction and gene targeting of patient-specific iPS cells}

Targeting plasmid was constructed using two-round recombination as previously reported. Freshly prepared BAC DNA containing the HBB locus was transformed into EL350 cells. pL253 plasmid containing the recombinant fragments was linearized by HindIII and electroporated into EL350 cells to generate the recombinant colonies. The fragment containing the Neo resistant gene was used for the second-round recombination.

Before electroporation, iPS cells were first adapted to enzymatically disaggregation into single cells using TrypLE select (Invitrogen) for several passages. On the day of electroporation, $1 \times 10^{7}$ iPS cells were suspended in ice-cold PBS containing $50 \mu \mathrm{g}$ linearized DNA and electroporated by electroporator (Gene Pulser II System, Bio-Rad) using the parameters $250 \mathrm{~V}$ and $500 \mu \mathrm{F}$. G418 selection started 5 days after electroporation with the concentration of $50 \mu \mathrm{g} / \mathrm{ml}$ and maintained for 5-7 days. Colonies were allowed to grow for an additional 7 days before picking.

\section{Southern blot analysis and DNA sequencing analysis}

For Southern blot analysis, genomic DNA $(30 \mu \mathrm{g})$ from the iPSCs was digested with $B g / I I$ overnight. Digested DNA fragments were separated on a $0.8 \%$ agarose gel and transferred to a nylon membrane (Amersham). The sequences of DNA probe primers are: F-GTAGAGGCTTGATTTGGAGGTT; R-TTATGGTGCTTCTGGCTCTG. The membrane was incubated with ${ }^{32} \mathrm{P}$-labeled DNA probe supplied by Amersham Rediprime II Random Prime Labeling System (GE Health). For sequencing analysis, the mutation site of $H B B$ gene were amplified by PCR from genomic DNA of piPSCs and ciPSCs and sequenced by BGI Life Tech company. The sequences of HBB sequencing primer are: F-CACTAGCAACCTCAAACAGACA; R-CTCAAGGCCCTTCATAATATCC.

\section{STR analysis}

For STR analysis, genomic DNA was extracted from 16 piPSC lines, 2 ciPSC lines and the patient fibroblast cells, respectively, and used as templates for PCR. Specific primers with 5' FAM were used in PCR system for STR D6S2427 cloning. STR signal analysis was performed by BGI Life Tech company.

\section{Hematopoietic differentiation}

For hES and iPS cell differentiation, OP9 cells were plated onto gelatinized $35-\mathrm{mm}$ or $100-\mathrm{mm}$ dishes in $\alpha$-MEM (Invitrogen) containing 20\% FBS (HyClone), 2 mM L-glutamax (Invitrogen) and $50 \mu \mathrm{g} / \mathrm{ml}$ penicillin/streptomycin (Millipore). After formation of confluent cultures on days 4 and 5 , half of the medium was changed, and cells were cultured for an additional 3 to 4 days. Undifferentiated hESCs and iPSCs were harvested by mechanical dissociation into small clumps. Both hESCs and iPSCs were added to OP9 cultures at a density of $1.5 \times 10^{6} / 20 \mathrm{ml}$ per 100 -mm dish 
or $0.3 \times 10^{6} / 4 \mathrm{ml}$ per 35 -mm dish in $\alpha$-MEM (Invitrogen) supplemented with $10 \%$ FBS (HyClone) and $100 \mu \mathrm{M}$ monothioglycerol (MTG; Sigma). The hESC/OP9 and iPSC/OP9 co-cultures were incubated for up to 10 days with a half-medium change every other day. Cells were harvested on day 10, and single-cell suspension was prepared for clonogenic and flow-cytometric assays.

\section{Flow cytometry analysis}

To analyze the phenotype of HPs, hESC/OP9 and iPSC/OP9 co-culture were collected and washed with FACS buffer (phosphate-buffered salt solution with $2 \%$ FBS). Cells were stained with anti-human CD43-PE monoclonal antibody (BD), anti-human CD31-APC monoclonal antibody (BD), anti-human CD45-PE/ Cy7 monoclonal antibody (BD), and anti-human CD235a-PE/ Cy5 monoclonal antibody (BD) with different combinations. Cells were then washed and resuspended in FACS buffer. Gating was done with matched isotype control monoclonal antibodies. 7-AAD (BD) was used to exclude dead cells. All analyses were performed on a MoFlo XDP cell sorter (Beckman Coulter Inc) running Summit software.

For analysis of human cell ratio, the peripheral blood cells were collected and the BM cells were flushed from the intra tibia of transplanted SCID mice. After washing with FACS buffer, cells were stained with anti-human HLA-ABC-FITC monoclonal antibody (BD), and incubated with Red Blood Cell Lysis Buffer (Tiagen) to remove the RBC, and then resuspended in FACS buffer and analyzed using MoFlo XDP cell sorter (Beckman Coulter Inc).

For analysis of human $\beta$-globin, the peripheral blood cells were collected. After fixation and permeabilization, cells were incubated with anti-human $\beta$-globin monoclonal antibody (Santa Cruz), and then washed with FACS buffer and analyzed by using MoFlo XDP cell sorter (Beckman Coulter Inc).

\section{Clonogenic progenitor cell assays}

Hematopoietic clonogenic assays were performed in 6-well ultralow attachment plates (Costa, Corning) using a $2 \mathrm{ml} /$ well of Methylcellulose-based media HSC003 (Stem Cell Technologies). Cells from hES cell/OP9 and iPS cell/OP9 co-cultures were collected, sorted by MACS and plated at $1 \times 10^{4} /$ well after 8 days of differentiation. All clonogenic progenitor assays were performed in triplicate. CFCs were scored after 14 days of incubation. Cytospin preparations from single colonies were made using a cytospin centrifuge, then fixed with methanol and stained with Wright stain (Sigma) to confirm the cell content of appropriate colonies.

\section{MACS analysis}

Cells from hESC/OP9 and iPSC/OP9 co-cultures were collected, washed with MACS buffer (phosphate-buffered salt solution with $0.5 \%$ BSA, and $2 \mathrm{mM}$ EDTA), and incubated with CD34 MicroBeads (Miltenyi Biotec) for magnetic labeling. Then cells were loaded onto the column. Magnetic separation was performed in the magnetic field following the manufacturer's instructions.

\section{BM transplantation}

Immune deficient NOD/SCID mice at the age of 8 to 10 weeks were sublethally irradiated with 3.0 Gry $6 \mathrm{~h}$ before transplantation. For transplantation, $5 \times 10^{5}$ MACS sorted CD $34^{+}$hematopoietic progenitors were collected as previously described and resuspended in IMDM medium, and then transferred to a $28 \mathrm{G}$ insulin syringe. The cell suspension in the syringe was gently inserted in a twisting motion vertically and was transplanted by intra-tibiae injection.

\section{HPLC}

RBC $(20 \mu \mathrm{l})$ from mouse peripheral blood were lysed in $500 \mu \mathrm{l}$ of lysis buffer (0.1 M 2-mercaptoethanol, $0.1 \mathrm{M} \mathrm{HCl})$, and then made up to $1 \mathrm{ml}$ with $500 \mu \mathrm{l}$ of $50 \%$ aqueous acetonitrile. The lysed red cells were centrifuged and the supernatant was analyzed by HPLC. Globin chain separation was performed by loading $30 \mu \mathrm{l}$ of lysed RBC onto a Vydac C4 column (Vydac, Hesperia, USA). At the start of each run, the buffer ratio between buffer A $(0.1 \%$ trifluoroacetic acid, Sigma) and buffer B (95\% acetonitrile, Sigma, $4.0 \%$ water, $0.1 \%$ trifluoroacetic acid) was $65 \%: 35 \%$. Buffer B was gradually increased to $56 \%$ after $1 \mathrm{~h}$ with a constant flow rate of $1 \mathrm{ml} / \mathrm{min}$. The proteins eluting from the column were measured at $220 \mathrm{~nm}, 280 \mathrm{~nm}$, and $413 \mathrm{~nm}$ with Agilent 1100 Diode Array Detector (Waters). Peak protein fractions were collected, and analyzed by tandem mass spectrometry or western blot using specific human $\beta$-globin (Santa Cruz) or $\gamma$-globin (Santa Cruz) antibody in order to confirm globin chain type.

\section{Microarray analysis}

Affymetrix GeneChip Human Genome U133 plus 2.0 Array (Affymetrix, Inc.) was used for microarray hybridizations to examine the global gene expression of hES cells, piPS7 cells, ciPS5 cells, and patient fibroblast cells. Briefly, total RNA was isolated using Trizol reagent (Invitrogen). cDNA was synthesized and amplified with WT cDNA Synthesis and Amplification Kit (Affymetrix, Inc.), labeled with biotin using WT Terminal Labeling Kit (Affymetrix, Inc.), hybridized to Affymetrix GeneChip Human Genome U133 plus 2.0 Array and analyzed by GeneChip Scanner 3000 7G3000 (Affymetrix, Inc.). All samples were prepared in three biological repeats. Data were analyzed using GCOS 1.4 software provided by Affymetrix. Signal values of probes presented in two samples were plotted in a scatter graph. Pearson's Correlation Coefficient $(\mathrm{R})$ between samples was calculated by Excel. We have submitted the microarray data to GEO database (GSE30220).

\section{Acknowledgments}

We are grateful to colleagues in our laboratory for their assistance with experiments and in the preparation of the manuscript. This project was supported by the Chinese Ministry of Science and Technology (2011CB964800, 2011ZX102-010, and 2010CB944900 to SG, and 2009AZ4006-F to ZY), and the "stem cell therapy for $\beta$-thalassemia" of Guangxi Zhuang Autonomous Region Science and Technology Bureau (GKZ0897009 to ZY).

\section{References}

1 Takahashi K, Yamanaka S. Induction of pluripotent stem cells from mouse embryonic and adult fibroblast cultures by defined factors. Cell 2006; 126:663-676.

2 Okita K, Ichisaka T, Yamanaka S. Generation of germlinecompetent induced pluripotent stem cells. Nature 2007; 448:313-317.

3 Wernig M, Meissner A, Foreman R, et al. In vitro reprogramming of fibroblasts into a pluripotent ES-cell-like state. $\mathrm{Na}$ - 
ture 2007; 448:318-324.

4 Takahashi K, Tanabe K, Ohnuki M, et al. Induction of pluripotent stem cells from adult human fibroblasts by defined factors. Cell 2007; 131:861-872.

5 Park IH, Zhao R, West JA, et al. Reprogramming of human somatic cells to pluripotency with defined factors. Nature 2008; 451:141-146.

6 Yu J, Vodyanik MA, Smuga-Otto K, et al. Induced pluripotent stem cell lines derived from human somatic cells. Science 2007; 318:1917-1920.

7 Dimos JT, Rodolfa KT, Niakan KK, et al. Induced pluripotent stem cells generated from patients with ALS can be differentiated into motor neurons. Science 2008; 321:1218-1221.

8 Ebert AD, Yu J, Rose FF Jr, et al. Induced pluripotent stem cells from a spinal muscular atrophy patient. Nature 2009; 457:277-280.

9 Raya A, Rodriguez-Piza I, Guenechea G, et al. Diseasecorrected haematopoietic progenitors from Fanconi anaemia induced pluripotent stem cells. Nature 2009; 460:53-59.

10 Soldner F, Hockemeyer D, Beard C, et al. Parkinson's disease patient-derived induced pluripotent stem cells free of viral reprogramming factors. Cell 2009; 136:964-977.

11 Hanna J, Wernig M, Markoulaki S, et al. Treatment of sickle cell anemia mouse model with iPS cells generated from autologous skin. Science 2007; 318:1920-1923.

12 Wang Y, Jiang Y, Liu S, Sun X, Gao S. Generation of induced pluripotent stem cells from human beta-thalassemia fibroblast cells. Cell Res 2009; 19:1120-1123.

13 Ye L, Chang JC, Lin C, Sun X, Yu J, Kan YW. Induced pluripotent stem cells offer new approach to therapy in thalassemia and sickle cell anemia and option in prenatal diagnosis in genetic diseases. Proc Natl Acad Sci USA 2009; 106:9826-9830.

14 Costa M, Dottori M, Sourris K, et al. A method for genetic modification of human embryonic stem cells using electroporation. Nat Protoc 2007; 2:792-796.

15 Liu P, Jenkins NA, Copeland NG. A highly efficient recombineering-based method for generating conditional knockout mutations. Genome Res 2003; 13:476-484.

16 Vodyanik MA, Slukvin II. Hematoendothelial differentiation of human embryonic stem cells. Curr Protoc Cell Biol 2007; Chapter 23:Unit 23.6.

17 Hockemeyer D, Soldner F, Beard C, et al. Efficient targeting of expressed and silent genes in human ESCs and iPSCs using zinc-finger nucleases. Nat Biotechnol 2009; 27:851-857.

18 Cui X, Ji D, Fisher DA, Wu Y, Briner DM, Weinstein EJ. Targeted integration in rat and mouse embryos with zinc-finger nucleases. Nat Biotechnol 2011; 29:64-67.

19 Song $\mathrm{H}$, Chung SK, Xu Y. Modeling disease in human ESCs using an efficient BAC-based homologous recombination system. Cell Stem Cell 2010; 6:80-89.

20 Liu GH, Suzuki K, Qu J, et al. Targeted gene correction of laminopathy-associated LMNA mutations in patient-specific
iPSCs. Cell Stem Cell 2011; 8:688-694.

21 Li M, Suzuki K, Qu J, et al. Efficient correction of hemoglobinopathy-causing mutations by homologous recombination in integration-free patient iPSCs. Cell Res 2011; 21:17401744.

22 Warren L, Manos PD, Ahfeldt T, et al. Highly efficient reprogramming to pluripotency and directed differentiation of human cells with synthetic modified mRNA. Cell Stem Cell 2010; 7:618-630.

23 Anokye-Danso F, Trivedi CM, Juhr D, et al. Highly efficient miRNA-mediated reprogramming of mouse and human somatic cells to pluripotency. Cell Stem Cell 2010; 8:376-388.

24 Miyoshi N, Ishii H, Nagano $\mathrm{H}$, et al. Reprogramming of mouse and human cells to pluripotency using mature microRNAs. Cell Stem Cell 2011; 8:633-638.

$25 \mathrm{Kim} \mathrm{D}$, Kim CH, Moon JI, et al. Generation of human induced pluripotent stem cells by direct delivery of reprogramming proteins. Cell Stem Cell 2009; 4:472-476.

26 Lister R, Pelizzola M, Kida YS, et al. Hotspots of aberrant epigenomic reprogramming in human induced pluripotent stem cells. Nature 2011; 471:68-73.

27 Ohi Y, Qin H, Hong C, et al. Incomplete DNA methylation underlies a transcriptional memory of somatic cells in human iPS cells. Nat Cell Biol 2011; 13:541-549.

28 Zhao T, Zhang ZN, Rong Z, Xu Y. Immunogenicity of induced pluripotent stem cells. Nature 2011; 474:212-215.

29 Papapetrou EP, Lee G, Malani N, et al. Genomic safe harbors permit high beta-globin transgene expression in thalassemia induced pluripotent stem cells. Nat Biotechnol 2011; 29:7378.

30 Zhou Q, Brown J, Kanarek A, Rajagopal J, Melton DA. In vivo reprogramming of adult pancreatic exocrine cells to betacells. Nature 2008; 455:627-632.

31 Vierbuchen T, Ostermeier A, Pang ZP, Kokubu Y, Sudhof TC, Wernig M. Direct conversion of fibroblasts to functional neurons by defined factors. Nature 2010; 463:1035-1041.

32 Huang $\mathrm{P}, \mathrm{He} \mathrm{Z}$, Ji S, et al. Induction of functional hepatocytelike cells from mouse fibroblasts by defined factors. Nature 2011; 475:386-389.

33 Ieda M, Fu JD, Delgado-Olguin P, et al. Direct reprogramming of fibroblasts into functional cardiomyocytes by defined factors. Cell 2010; 142:375-386.

34 Szabo E, Rampalli S, Risueno RM, et al. Direct conversion of human fibroblasts to multilineage blood progenitors. Nature 2010; 468:521-526.

35 Agarwal S, Loh YH, McLoughlin EM, et al. Telomere elongation in induced pluripotent stem cells from dyskeratosis congenita patients. Nature 2010; 464:292-296.

36 Marion RM, Strati K, Li H, et al. Telomeres acquire embryonic stem cell characteristics in induced pluripotent stem cells. Cell Stem Cell 2009; 4:141-154.

(Supplementary information is linked to the online version of the paper on the Cell Research website.) 